\title{
New Planetary Nebulae found by the IPHAS Survey
}

\author{
K. Viironen ${ }^{1}$, L. Sabin ${ }^{2}$, E. R. Rodríguez-Flores ${ }^{3,1}$, A. Mampaso ${ }^{1}$, \\ R. L. M. Corradi ${ }^{2,1}$ and R. Greimel ${ }^{2}$ \\ ${ }^{1}$ Instituto de Astrofísica de Canarias, C/Vía Láctea s/n, E-38200 La Laguna, Tenerife, Spain \\ e-mail: kerttu@iac.es, amr@iac.es \\ ${ }^{2}$ Isaac Newton Group of Telescopes, Apartado de Correos 321, E-38700 Sta. Cruz de La \\ Palma, Spain \\ e-mail: lsabin@ing.iac.es, rcorradi@ing.iac.es, greimel@ing.iac.es \\ ${ }^{3}$ Instituto de Geofísica y Astronomía, Calle 212 No. 2906, CP 11600, La Habana, Cuba \\ e-mail: erflores@iga.cu
}

\begin{abstract}
We are currently undertaking a systematic search of new Galactic planetary nebulae (PNe) in the IPHAS (INT Photometric H-Alpha Survey) data. We employ two methods to search for PNe of different sizes: a visual search through continuum subtracted H-Alpha mosaics for extended PNe and a (point) source catalog based search for small diameter PNe. Here we present the methodology and first results of the small diameter PNe search.
\end{abstract}

Keywords. planetary nebulae, abundances

IPHAS is currently mapping 1800 degrees $^{2}$ of the Northern Galactic plane (a band between $\mathrm{b}=-5$ to +5 degrees) in three filters using the INT Wide Field Camera at the Observatorio del Roque de los Muchachos (La Palma, Spain). The survey is an international collaboration of 15 institutes led by J. Drew at Imperial College (London, UK). IPHAS observing started in August 2003 and is expected to be completed by the end of 2007, with an estimated total of 30 observing weeks (mostly bright nights). A narrowband $\mathrm{H} \alpha$ and two Sloan filters (r' and i') are used for matched 120, 30, and $10 \mathrm{~s}$ exposures, respectively, spanning the magnitude range $r^{\prime}=13$ to 20 mag for point sources. IPHAS is the first fully-photometric $\mathrm{H} \alpha$ survey of the Galactic plane. It will discover around 40,000 new emission-line stars, including young stars ( T Tau, Herbig AeBe stars, etc.), evolved stars (post-AGB, LBVs, etc.) as well as binaries (CVs, symbiotic stars, etc.) in addition to thousands of extended nebulae such as PNe, H-H objects, HII regions, SN remnants, etc. More information about the survey can be found in Drew et al. (2005) and in the official survey website, http://www.iphas.org/.

Two systematic searches for PNe are currently conducted on the IPHAS data. Extended PNe are searched visually from continuum subtracted mosaics of IPHAS data while the compact PNe search is based on IPHAS catalog information (see Corradi et al. 2005). The first step to select compact PN candidates is to filter out all objects which are too close to the borders of the CCDs or to the areas of bad pixels as well as sources which are not classified as stars or extended objects. Also a first cut in the r' $-\mathrm{H} \alpha$ plane is made removing all objects with $\mathrm{r}^{\prime}-\mathrm{H} \alpha<(\mathrm{r}-\mathrm{H} \alpha)_{\text {median }}+1$, which removes the majority of the stellar locus objects (see Fig. 1, Left). We also remove sources were the positional match between individual images is worse than 0.65 arcsec.

The 2003 data contains a total of 475 million catalogued objects, of which 368961 passed the initial selection. After an additional sub-selection of objects with $\mathrm{r}-\mathrm{H} \alpha>$ 2.0 or $1.5<\mathrm{r}-\mathrm{H} \alpha<2.0$ and $\mathrm{H} \alpha<17 \mathrm{mag}$, there remained 934 objects. These objects 

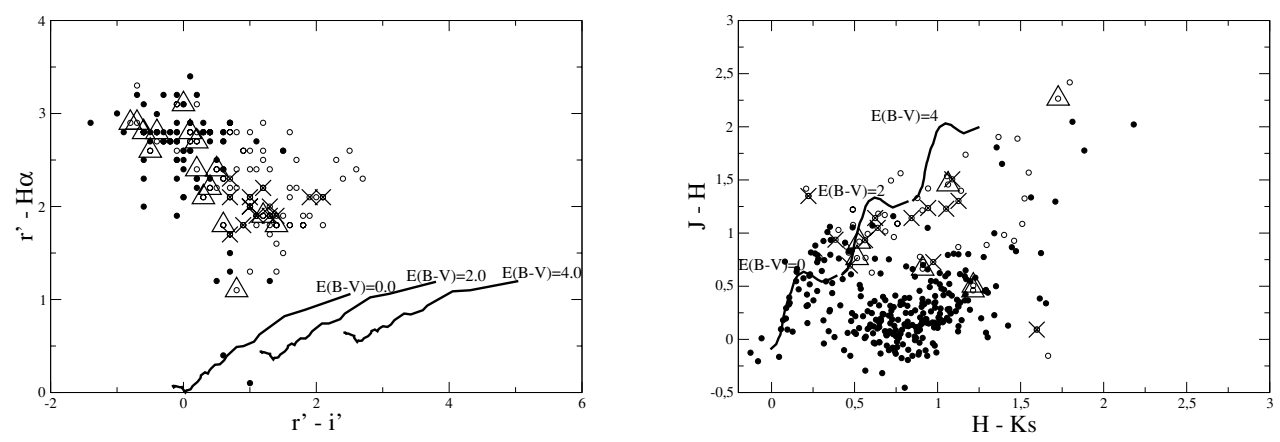

Figure 1. Left: The location of known PNe (filled circles), candidate PNe (open circles), confirmed PNe (triangles) and confirmed $\mathrm{H} \alpha$ emission-line stars (crosses) in the IPHAS two-color diagram. Also the locations of main sequence stars with different reddenings are shown (solid black lines, from Drew et al. 2005). Right: 2MASS color-color diagram. The symbols are the same as in the figure on the left. Data for known PNe are taken from Larios \& Phillips (2005).

were then visually checked to remove contaminating sources like HII region condensations. In the end 44 candidates were left. Adding part of the 2004 data more than a hundred small diameter PNe candidates are found and the search is continuing. Together with the visual search for extended PNe we expect to find a total of 500-1000 new Galactic PNe.

The use of the 2MASS $(\mathrm{J}-\mathrm{H}) /(\mathrm{H}-\mathrm{Ks})$ color-color diagram as an additional tool for finding PNe has also been studied. Figure 1 (Right) represents the known PNe, IPHAS candidate PNe, confirmed new IPHAS PNe and new IPHAS $\mathrm{H} \alpha$ emission-line stars in the 2MASS two-color diagram. The IPHAS and 2MASS objects were assumed to be the same if the difference between the coordinates was $<1$ arcsec. The combination of IPHAS and 2MASS colors can be used to separate PNe from other stars.

The follow up spectroscopy of the candidate PNe is continuing. Spectra of 35 compact $\mathrm{PNe}$ candidates have been obtained so far with the $2.1 \mathrm{~m}$ OAN telescope at San Pedro Mártir, México and with the $4.2 \mathrm{~m}$ WHT+ISIS at Roque de los Muchachos, La Palma, Spain. 17 of them have been confirmed to be PNe and the remaining 18 candidates are emission-line stars. One of the new PNe has a very large galactocentric distance $(14-20 \mathrm{kpc})$, being very likely the most distant Galactic PN with measured chemical abundances (Mampaso et al. 2005).

\section{References}

Corradi, R.L.M, Mampaso, A., Viironen, K. et al. 2005, Planetary Nebulae as Astronomical Tools Proc. AIP Conference No 804, p. 7

Drew, J.E., Greimel, R., Irwin M.J. et al. 2005, MNRAS 362, 753

Larios, G.R. \& Phillips, J.P. 2005, MNRAS 357, 732

Mampaso, A., Viironen, K., Corradi, R.L.M. et al. 2005, Planetary Nebulae as Astronomical Tools Proc. AIP Conference No 804, p. 14 